\title{
Comparison of Menstrual Effluent Vascular Endothelial Growth Factor Immunocytochemistry Expression Between Endometriosis and Non-Endometriosis Patients
}

\author{
Ruswana Anwar ${ }^{*}$, Sunardi', Siti Salima ${ }^{1}$, Setyorini Irianti', Benny Hasan Purwara', Tono \\ Djuwantono ${ }^{1}$, Wiryawan Permadi ${ }^{1}$, Tita Husnitawati Madjid ${ }^{1}$
}

\begin{abstract}
Objectives: This study aimed to measure the vascular endothelial growth factor (VEGF) expression in menstrual effluent from patients with endometriosis compared to non-endometriosis through immunocytochemical methods. We also measured the degree of pain level, endometrioma cyst size, and infertility status whether it is affected by VEGF expression.

Materials and Methods: The present case-control study was conducted in Hasan Sadikin General Hospital, Bandung. Thirty productive-age women diagnosed with endometrioma and 30 productive-age women without endometriosis as the control group were included in this study. Menstrual effluent was taken from the posterior fornix on the second day of menstruation and stained using immunocytochemistry staining for VEGF.

Results: The results demonstrated a significant difference between the two study groups in terms of VEGF intensity and histoscore although no difference was found in VEGF distributions between the study groups. The subjects in the endometriosis group had significantly higher VEGF intensity and significantly higher VEGF histoscore compared to the control group. Women with VEGF histoscore more than 6 has 9.33 times risk of developing endometriosis compared to those with lower histoscore. There were no significant correlations between VEGF and pain scale, infertility, and the cyst size. Finally, the cyst size was proportionally related to pain.

Conclusions: VEGF distribution and expression in endometriosis women were significantly higher than VEGF levels in nonendometriosis women. Women with menstrual effluent containing higher VEGF levels had more chances of developing endometriosis compared to those with lower VEGF levels. Eventually, larger endometrioma size was proportionally related to higher pain levels in subjects with endometrioma.

Keywords: Endometriosis, VEGF, Pain, Cyst size, Infertility
\end{abstract}

\section{Introduction}

Endometriosis is a disease characterized by the presence of endometrial glands and endometrial stroma in ectopic locations outside the uterus, especially in the pelvic, ovarian, and rectovaginal septum. This disease affects around $10 \%$ of all premenopausal women worldwide although only around $30-40 \%$ are experiencing the symptoms. The point prevalence of endometriosis was 10.8 per 1000 women. The highest age group experiencing endometriosis includes 40-44 year-old women.

The etiopathogenesis of endometriosis is not yet fully understood, but several theories have emerged that explain the pathogenesis of the disease. One theory that has been long known since around 1920 is the theory of retrograde menstruation (Sampson's Theory). It explains that women suffering from endometriosis experience retrograde menstruation, resulting in the reflux of endometrial cells into the peritoneal cavity. This phenomenon occurs through a patent fallopian tube (1).
Recent studies have found that oxidative stress and proinflammatory mediators are related to the development and progression of endometriosis. The activity of macrophages and mononuclear cells in the peritoneal fluid will increase the production of reactive oxygen species (ROS), triggering the progression of endometriosis. The increased ROS production occurring in the body cannot be balanced by antioxidants, which have a role as a pro-inflammatory cytokine eliminator in the human body. Oxidative stress may be used to determine the extent and depth of endometriotic lesions. In addition, evaluating women with endometriosis, Gupta et al found an increase in oxidative stress in the abdominal cavity. Pro-inflammatory factors will trigger the occurrence of oxidative stress. One of the pro-inflammatory factors is cellular debris which acts as a triggering component of oxidative stress production. Systemic effects can occur due to the entry of cellular debris into the plasma stream (2).

Angiogenesis is a factor that is known to have 
Key Messages

- Women with endometriosis had significantly higher VEGF intensity and histoscore compared to healthy women.

- There were no significant correlations between VEGF and pain scale, infertility, and the cyst size.

an important role in the pathological process of endometriosis. One of the main mediators of angiogenesis is vascular endothelial growth factor (VEGF), which is a vasoactive substance that is involved in various physiological processes in the body, including the process of wound healing and endometrial revascularization. The activation of VEGF receptors in the endothelial cells of the blood vessel by VEGF signals will mediate the process of the proliferation and migration of endothelial cells, increase vascular permeability, and induce the vascular remodeling process, leading to the formation of new blood vessels. Although VEGF is known to be naturally produced by tissues in the female genital tract (e.g., the endometrium, ovaries, and placenta), VEGF receptors are only found in endothelial cells. Estradiol has been known to regulate VEGF expression. Increased estradiol can cause an increase in VEGF mRNA expression in the functional layer of the endometrium.

\section{Materials and Methods}

This observational-analytic study with a comparative casecontrol study design was conducted at Dr. Hasan Sadikin General Hospital Bandung from May to December 2019.

The included subjects in the endometriosis group were married women of reproductive age who were in the age range of 20-42 years old and diagnosed with endometrioma through ultrasound examinations confirmed by the clinical diagnosis of endometriosis after surgery and pathological slide examinations. On the other hand, patients were excluded if they used contraception for at least 3 months before the surgery, were using gonadotropin-releasing hormone analog medicines, had an implant or intrauterine device containing levonorgestrel for at least 6 months before the surgery, and had an infection disease/malignancy.

Those in the control group were female patients aged 20-42 years who came to Aster Fertility Clinic at Dr. Hasan Sadikin having had a normal uterus and ovaries based on ultrasound examinations and a regular menstrual cycle for the last three months. Patients later identified as experiencing endometriosis or endometriosis-like symptoms were excluded from the control group.

Patients underwent gynecologic check-ups or examinations at the Obstetrics and Gynecology Clinic, Hasan Sadikin Hospital Bandung diagnosed with endometriosis were asked whether they were willing to join this study. If they showed their willingness, they were asked to sign an informed consent letter and were scheduled for menstrual blood sample taking. The date for menstrual blood sample aspiration were collected two days after the first day of the period (e.g., the third day of the period). The subjects of the control group also had the same sampling procedure, the only difference was that they were normal patients without any endometriosis symptoms. We asked both groups regarding their infertility status. Infertility in this study was defined as the inability of a couple to conceive an offspring after one year of routine copulation with normal frequency.

Menstrual effluent samples are taken from the posterior fornix using a syringe, processed in a laboratory, and placed on an object glass for further staining according to the immunocytochemical staining procedure with the VEGF stain. The immunocytochemical assessment in this study was carried out by looking at the color intensity of endometrial cells found in subjects' menstrual effluent samples after immunocytochemical staining for VEGF. Positive VEGF colored cells will appear as brownish cells. The positive cells stained by the VEGF stain are then measured for distribution and intensity. Cell distribution was assessed in 5 fields of distributing the cells, counting the cells that become brown due to the staining, determining its distribution, and then classifying the field of view into several groups ( $<20 \%, 20-50 \%, 50-80 \%$, $\&>80 \%$ ), and then measuring the intensity of staining. The intensity of VEGF staining was qualitatively assessed looking at the level of the staining of VEGF cells, which were included as the scoring.

The data were analyzed by SPSS for Windows, version 21. A normality test for numeric variables was done using the Shapiro-Wilk test. The Chi-square and Kruskal-Wallis statistical tests were used for the analysis. The Chi-square test was applied to measure the value difference between the groups, and the Kruskal-Wallis test was used to analyze the relationship between the cyst size and pain scale in subjects with endometrioma. The cut-off point was analyzed using the receiver operating characteristics (ROC) curve.

\section{Results}

A total of 60 women participated in this study. The demographic characteristics of the patients are listed in Table 1. The age range of subjects with endometriosis and non-endometriosis was 23-42 and 20-42 years, respectively. There were 11 nulliparous subjects, 12 subjects with single parity, and 7 subjects with two or more parity in the endometriosis group. In the nonendometriosis group, there were 5 nulliparous subjects, 13 subjects with single parity, and 12 subjects with two or more parity. The average body mass index (BMI) was $23.6 \mathrm{~kg} / \mathrm{m}^{2}$ and $22.0 \mathrm{~kg} / \mathrm{m}^{2}$ in the endometriosis and nonendometriosis groups, respectively. There were 11 infertile and 19 fertile subjects in the endometriosis group whereas 5 infertile and 19 fertile subjects in the non-endometriosis group. Based on the statistical analysis, there was no 
Table 1. Characteristics of Research Subjects

\begin{tabular}{|c|c|c|c|}
\hline \multirow[b]{2}{*}{ Characteristics } & \multicolumn{2}{|c|}{ Groups } & \multirow[b]{2}{*}{$P$ Value } \\
\hline & $\begin{array}{c}\text { Endometriosis } \\
n=30\end{array}$ & $\begin{array}{c}\text { Non-Endometriosis } \\
\mathrm{n}=\mathbf{3 0}\end{array}$ & \\
\hline \multicolumn{4}{|l|}{ Age $(y)$} \\
\hline$<25$ & 3 & 6 & \multirow{5}{*}{$0.167^{a}$} \\
\hline $25-34$ & 11 & 15 & \\
\hline$\geq 35$ & 16 & 9 & \\
\hline Average (SD) & $34.4(6.0)$ & $31.1(6.1)$ & \\
\hline Range & $23-42$ & $20-42$ & \\
\hline \multicolumn{4}{|l|}{ Parity } \\
\hline 0 & 11 & 5 & \multirow{3}{*}{$0.165^{\mathrm{a}}$} \\
\hline 1 & 12 & 13 & \\
\hline$\geq 2$ & 7 & 12 & \\
\hline $\mathrm{BMI}\left(\mathrm{kg} / \mathrm{m}^{2}\right)$ & & & $0.055^{\mathrm{b}}$ \\
\hline Mean (SD) & $23.6(3.4)$ & $22.0(3.0)$ & \\
\hline Range & $17.5-32.9$ & $16.4-29.4$ & \\
\hline Infertility status & & & $0.080^{\mathrm{a}}$ \\
\hline Yes & 11 & 5 & \\
\hline No & 19 & 25 & \\
\hline
\end{tabular}

Note. SD, standard deviation; BMI, body mass index.

${ }^{\text {a }}$ Based on chi-square test; ${ }^{\mathrm{b}}$ Based on the independent $t$ test.

characteristic difference between these two groups.

VEGF intensity measurement showed that there were significant differences between the two groups $(P<0.05)$. In endometriosis subjects, the intensity of VEGF tends to be stronger (56.7\%) whereas it tends to be moderate (33.3\%) in non-endometriosis subjects. The distribution of VEGF in the two study groups demonstrated no significant difference $(P>0.05)$ between the two groups. Based on histoscore calculations, a median value of 4 and 3 was obtained for subjects with endometriosis and nonendometriosis subjects, respectively, and this difference was statistically significant $(P<0.05)$. This indicates that VEGF expression in menstrual effluent endometrial cells with endometriosis patients is significantly stronger than menstrual effluent in non-endometriosis subjects, as evidenced by the results of statistical tests. The related data are shown in Table 2.

Based on the results of VEGF intensity examination in the endometriosis group, 7, 6, and 17 subjects had weak, moderate, and strong VEGF intensity, respectively. In the non-endometriosis group, 2, 12, 10, and 6 subjects had negative, weak, moderate, and strong VEGF intensity, respectively. According to the results of the VEGF distribution in the endometriosis group, 13, 4, 4, and 9 subjects had a VEGF distribution of less than 20\%, $20-50 \%, 50-80 \%$, and over $80 \%$, respectively. In nonendometriosis subjects, $2,15,7,4$, and 2 subjects had a negative, less than $20 \%, 20 \%-50 \%, 50 \%-80 \%$, and more than $80 \%$, respectively. Based on histoscore calculations, 12 and 18 subjects had high and low VEGF expression, in the endometriosis group, respectively whereas in the non-
Table 2. Comparison of VEGF Intensity, Distribution, and Histoscore Between Endometriosis and Non-endometriosis Subjects

\begin{tabular}{|c|c|c|c|}
\hline \multirow[b]{2}{*}{ VEGF } & \multicolumn{2}{|c|}{ Groups } & \multirow[b]{2}{*}{$P$ Value } \\
\hline & $\begin{array}{c}\text { Endometriosis } \\
\quad(n=30)\end{array}$ & $\begin{array}{c}\text { Non-Endometriosis } \\
(n=30)\end{array}$ & \\
\hline \multicolumn{4}{|l|}{ Intensity } \\
\hline Negative (score: 0) & 0 & 2 & \multirow[t]{4}{*}{$0.035^{\mathrm{a}}$} \\
\hline Weak (score: 1) & 7 & 12 & \\
\hline Moderate (score: 2) & 6 & 10 & \\
\hline Strong (score: 3 ) & 17 & 6 & \\
\hline \multicolumn{4}{|l|}{ Distribution } \\
\hline Negative (score: 0) & 0 & 2 & \multirow{5}{*}{$0.388^{\mathrm{a}}$} \\
\hline$<20 \%$ (score: 1 ) & 13 & 15 & \\
\hline $20-50 \%$ (score: 2 ) & 4 & 7 & \\
\hline $50-80 \%$ (score: 3 ) & 4 & 4 & \\
\hline$>80 \%$ (score: 4 ) & 9 & 2 & \\
\hline \multicolumn{4}{|l|}{ Histoscore } \\
\hline Median & 4 & 3 & \multirow[t]{2}{*}{$0.020^{\mathrm{b}}$} \\
\hline Range & $1-12$ & $0-12$ & \\
\hline
\end{tabular}

Note. VEGF: Vascular endothelial growth factor.

${ }^{\mathrm{a}}$ Based on chi-square test; ${ }^{\mathrm{b}}$ Based on the independent $t$ test.

endometriosis group, 2 and 28 subjects had high and low VEGF expression, respectively. The VEGF expression is shown in Figures 1-4.

According to the analysis results of the comparison of VEGF histoscore between endometriosis and nonendometriosis cases that are statistically significant, the level of VEGF expression can be determined based on the ROC curve, which was made by linking 1-specificity with the sensitivity of various histoscores (Figure 5). The results of the curve show that there is a cut-off value of VEGF, which was shown to be more than 6 points as its threshold value with a sensitivity of $40 \%$ and a specificity of $93.33 \%$ $(P=0.013)$. There was a significant relationship between VEGF expression and the incidence of endometriosis $(P<0.05)$ with the magnitude of the odds ratio $(95 \%$ confidence interval: $9.33,1.87-46.48)$. The results of these calculations indicated that women with high VEGF expression (>6) had a risk of having endometriosis 9.33 times higher when compared with women who had low VEGF expression $(\leq 6)$. The data related to this finding are provided in Table 3.

Based on the obtained data (Table 4), the statistical analysis on the correlation between VEGF expression and pain scale demonstrated no significant correlation $(P>0.05)$. Similar results were also found in the correlation between VEGF expression and cyst size, indicating no significant correlation $(P>0.05)$ between these two variables (Table 5).

In this study, the size of the cyst increased as the pain scale represented an increase. Based on data (Table 6), the difference in the pain scale was statistically significant based on the Kruskal-Wallis test $(P<0.05)$ with a significant 
(A)

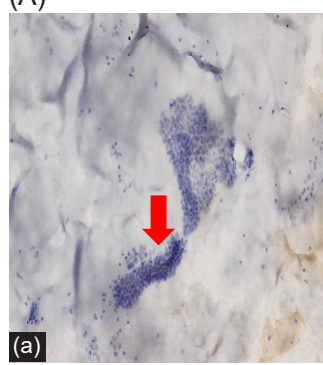

(B)

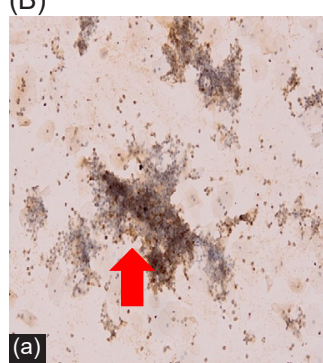

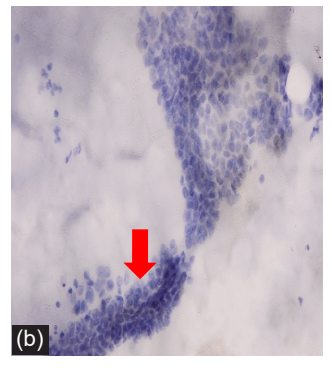

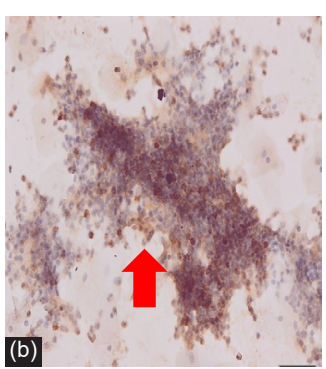

(C)

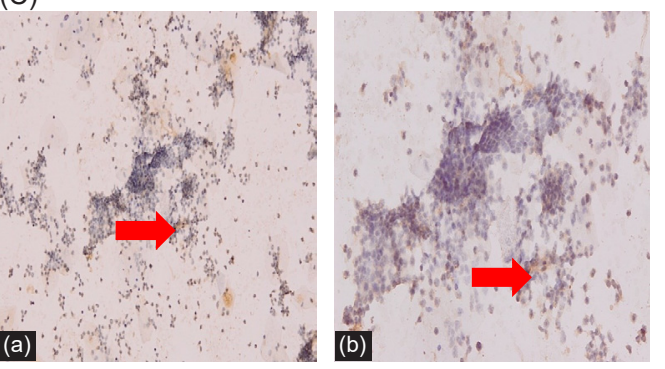

(D)

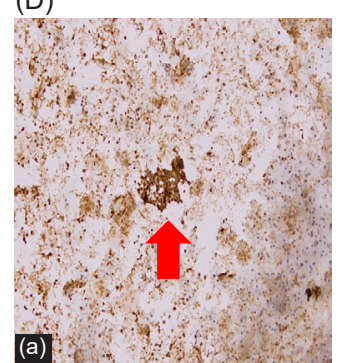

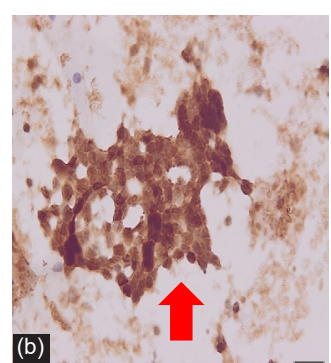

Figure 1. VEGF Staining Expression of Eutopic Menstrual Blood Endometrium Cells. (A) Negative appearance, (B) weak appearance, (C) Moderate appearance and (D) strong appearance. Note. VEGF: Vascular endothelial growth factor; (a) $\times 100$ magnification; (b) $\times 200$ magnification.

cyst size comparison based on the Mann-Whitney test for every study groups between mild and moderate pain scales $(P=0.003)$, mild and severe pain scale $(P=0.004)$, and moderate and severe pain scale $(P=0.048)$.

The results revealed that 3 and 8 subjects with high and low VEGF expression experienced and infertility,

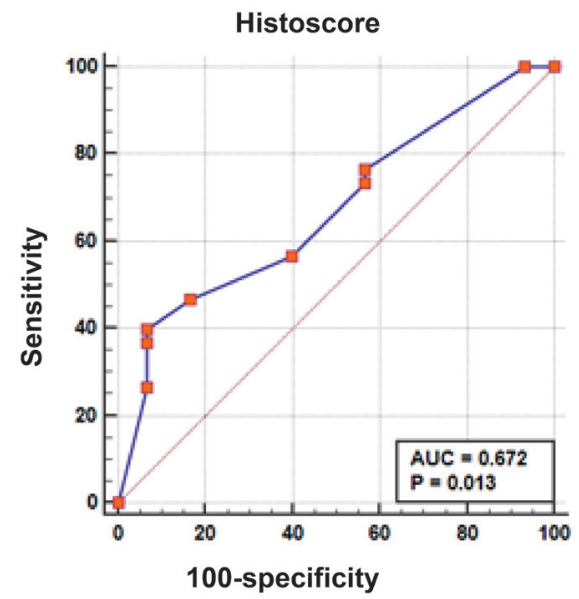

Figure 5. Cut-off Value of VEGF in Predicting the Incidence of Endometriosis Note. VEGF: Vascular endothelial growth factor. respectively (Table 7). Based on the results of Fisher's exact test, there was no correlation $(P>0.05)$ between VEGF expression and infertility status.

The results further demonstrated that the average size of the endometrial cyst in the infertile and non-infertile groups was 8.42 and $8.38 \mathrm{~cm}$, respectively (Table 8). However, no significant difference $(P>0.05)$ was found in the independent $\mathrm{t}$-test comparison between these two groups.

Regarding the correlation between infertility status and VEGF expression, the average cyst size of infertile and fertile subjects with high and low VEGF expression was 6.93 and 8.93, as well as 8.98 and $7.89 \mathrm{~cm}$, respectively (Table 9). There was no significant difference $(P>0.05)$ in the results of the independent $t$ test between these two groups.

\section{Discussion}

In this study, the mean age of the endometriosis and non-endometriosis groups was 34.4 and 31.1 years old, respectively. In the endometriosis group, most subjects were categorized into the age range of more than 35 years. This figure is more or less consistent with the results of previous studies on endometriosis in Indonesia (3). The

Table 3. The Relationship of VEGF Expressions With the Event of Endometriosis

\begin{tabular}{|c|c|c|c|c|}
\hline \multirow{2}{*}{ VEGF Expressions } & \multicolumn{2}{|c|}{ Study Groups } & \multirow[b]{2}{*}{$P$ Value $^{\text {a }}$} & \multirow[b]{2}{*}{ OR $(95 \% \mathrm{Cl}$} \\
\hline & Endometriosis $(n=30)$ & Non-endometriosis $(n=30)$ & & \\
\hline$>6$ (High) & $12(40 \%)$ & $2(6.7 \%)$ & \multirow{2}{*}{0.002} & $9.33(1.87-$ \\
\hline$\leq 6($ Low $)$ & $18(60 \%)$ & $28(93.3 \%)$ & & 46.48) \\
\hline
\end{tabular}

Note. VEGF: Vascular endothelial growth factor; OR: odds ratio; $\mathrm{Cl}$ : confidence interval.

${ }^{a}$ The chi-square test. 
Table 4. Correlation of VEGF Expression and Pain Scale in Patients With Endometriosis

\begin{tabular}{lccc}
\hline \multirow{2}{*}{ Pain Scale (VAS) } & \multicolumn{2}{c}{ VEGF Expression } & \multirow{2}{*}{ P Value $^{\mathrm{a}}$} \\
\cline { 2 - 3 } & High $(\mathbf{n}=\mathbf{1 2})$ & Low $(\mathbf{n}=\mathbf{1 8})$ & \\
\hline Mild & $3(25 \%)$ & $6(33 \%)$ & \\
Moderate & $7(58 \%)$ & $9(50 \%)$ & 0.878 \\
Severe & $2(17 \%)$ & $3(17 \%)$ & \\
\hline
\end{tabular}

Note. VEGF: Vascular endothelial growth factor; VAS: Visual analog scale. ${ }^{\text {a }}$ Based on chi-square test.

Table 5. Correlation of VEGF Expression and Cyst Size in Patients With Endometriosis

\begin{tabular}{lccc}
\hline \multirow{2}{*}{ Cyst Size (cm) } & \multicolumn{2}{c}{ VEGF Expression } & \multirow{2}{*}{ P Value $^{\mathbf{a}}$} \\
\cline { 2 - 3 } & High $(\mathbf{n}=\mathbf{1 2})$ & Low $(\mathbf{n}=\mathbf{1 8})$ & \\
\hline Average (SD) & $8.43(3.07)$ & $8.38(3.69)$ & \multirow{2}{*}{0.969} \\
Range & $4.14-1 \mathrm{~s} 4.29$ & $4.00-15.42$ & \\
\hline
\end{tabular}

Note. VEGF: Vascular endothelial growth factor; SD: Standard deviation.

${ }^{\text {a }}$ Based on chi-square test.

Table 6. Relationship Between Cyst Size and Pain Scale in Subjects With Endometrioma

\begin{tabular}{lcccc}
\hline \multirow{2}{*}{ Cyst Size $(\mathbf{c m})$} & \multicolumn{3}{c}{ Pain Scale } & \\
\cline { 2 - 4 } & $\begin{array}{c}\text { Mild } \\
(\mathbf{n}=\mathbf{9})\end{array}$ & $\begin{array}{c}\text { Medium } \\
(\mathbf{n = 1 6 )}\end{array}$ & $\begin{array}{c}\text { Weight } \\
(\mathbf{n}=\mathbf{5}\end{array}$ & V Value $^{\mathrm{a}}$ \\
\hline Average (SD) & $5.57(2.09)$ & $8.89(3.1)$ & $11.89(2.22)$ & \\
Median & 5 & 7.66 & 11.39 & 0.001 \\
Range & $4-10.42$ & $5.34-14.29$ & $9.32-15.42$ & \\
\hline
\end{tabular}

SD: Standard deviation. ${ }^{a}$ Based on The Kruskal-Wallis test.

Table 7. Correlation Between VEGF Levels and Infertility in Endometriosis Subjects

\begin{tabular}{lccc}
\hline \multirow{2}{*}{ Infertility Status } & \multicolumn{2}{c}{ VEGF Expression } & \multirow{2}{*}{ Value $^{\mathrm{a}}$} \\
\cline { 2 - 3 } & High $(\mathbf{n}=\mathbf{1 2})$ & Low $(\mathbf{n = 1 8})$ & \\
\hline Yes (infertile) & $3(25 \%)$ & $8(44 \%)$ & \multirow{2}{*}{0.442} \\
No (fertile) & $9(75 \%)$ & $10(55.6 \%)$ & \\
\hline
\end{tabular}

Note. VEGF: Vascular endothelial growth factor; SD: Standard deviation.

${ }^{a}$ Based on Fisher exact test.

Table 8. Comparison of Cyst Size According to Infertility Status on Endometriosis Patients

\begin{tabular}{lccc}
\hline \multirow{2}{*}{ Cyst Size $(\mathbf{c m})$} & \multicolumn{2}{c}{ Infertility Status } & \multirow{2}{*}{$\boldsymbol{P}$ Value $^{\mathbf{a}}$} \\
\cline { 2 - 3 } & Yes $(\mathbf{n}=\mathbf{1 1})$ & No $(\mathbf{n}=\mathbf{1 9})$ & \\
\hline Average (SD) & $8.42(3.87)$ & $8.38(3.21)$ & \multirow{2}{*}{0.977} \\
Range & $4.0-15.42$ & $4.0-14.29$ & \\
\hline
\end{tabular}

Note. SD: Standard deviation.

a Based on independent $t$ test.

results in this study are similar to those of the research conducted at Professor RD Kandou General Hospital Manado, where the findings demonstrated that the highest prevalence of endometrioma cases happened in the age group of more than 39 years. The mean age in this case was similar to a study performed at Cipto Mangunkusumo Hospital in Jakarta (4), reporting that the average age of endometrioma patients was 33.39 years and most
Table 9. Comparison of Cyst Size According to Infertility Status and VEGF Expression on Endometriosis Patients

\begin{tabular}{lccc}
\hline \multirow{2}{*}{ Cyst Size $(\mathbf{c m})$} & \multicolumn{2}{c}{ Infertility Status } & \multirow{2}{*}{ PValue $^{\mathrm{a}}$} \\
\cline { 2 - 3 } & Yes $(\mathbf{n}=\mathbf{1 1})$ & No $(\mathbf{n}=\mathbf{1 9})$ & \\
\hline High VEGF & $(\mathrm{n}=3)$ & $(\mathrm{n}=9)$ & \\
Average (SD) & $6.93(0.58)$ & $8.93(3.42)$ & 0.352 \\
Range & $6.5-7.59$ & $4.14-14.29$ & \\
\hline Low VEGF & $(\mathrm{n}=8)$ & $(\mathrm{n}=10)$ & \\
Average (SD) & $8.98(4.47)$ & $7.89(3.10)$ & 0.550 \\
Range & $4.0-15.42$ & $4.0-14.21$ & \\
\hline
\end{tabular}

Note. VEGF: Vascular endothelial growth factor; SD: Standard deviation. *Based on an independent $t$ test.

subjects were in the age group of 30-34 years old. Similar results were found in a study of endometriosis conducted at Dr. Soetomo Hospital in Surabaya, indicating that endometriosis was prevalent in the age of 30-35 years (5).

In the population of reproductive age women, the incidence of endometriosis was found to be $5-15 \%$ and is often found incidentally in women without the clinical symptoms of endometriosis $(6,7)$. Based on the results of a large-scale study conducted in Germany (8), the majority of endometriosis cases (80.36\%) occurred in premenopausal age (under 45 years). Around $17.09 \%$ of endometriosis patients were in the perimenopause group (45-55 years) and the remaining $2.55 \%$ of patients were in the post-menopausal age group (over 55 years). The group suffering from endometriosis belonged to the age group of 35-40 years, followed by the age groups of 4045 and 30-35 years. The remaining age groups suffering from endometriosis were in the range of 45-50 and 25-30 years. The authors suggested that the cases of pelvic pain without a clear pain source in women aged over 40 years could possibly be endometriosis due to the high number of endometriosis cases in this age group.

The prevalence rate of endometriosis is higher in Asian populations compared to Caucasian populations. The incidence of endometriosis in Asian populations was reported to be the highest among Chinese and Indians compared to other populations. According to evidence, the mean \pm standard deviation of the age of patients with endometriosis was as $35.79 \pm 3.70$ years. Asian women diagnosed with endometriosis were at a younger age and had a lower BMI compared to the Caucasian population (9).

Subjects in the non-endometriosis group were more likely to have two or more parity status although no difference was found between the parity status of endometriosis and non-endometriosis groups, which is in line with the results of Parasar et al, demonstrating that endometriosis was inversely related to parity and BMI (6). The increased parity is correlated with a decrease in endometriosis risk due to a decrease in the total amount of the menstrual cycle, which reduces the risk of menstrual effluent reflux to the peritoneal cavity. In this context, 
nulliparity or a decrease in parity might be considered as one of the early signs of endometriosis (10).

The average BMI in the endometriosis and nonendometriosis groups was $23.6 \mathrm{~kg} / \mathrm{m}^{2}$ and $22.0 \mathrm{~kg} / \mathrm{m}^{2}$, respectively. There was no statistically significant difference between the BMI in both groups, which contradicts the findings of Parasar et al and Shah et al, indicating an inverse correlation between BMI and endometriosis $(6,11)$. This hypothesis is based on the fact that obese women often experience amenorrhea or oligomenorrhea episodes, and are also related to the higher chance of obese woman to exhibit polycystic ovary syndrome. This syndrome causes anovulation, which can reduce retrograde menstruations and causes excess androgen hormones inhibiting the growth of endometriosis (11).

In this study, patients in the endometriosis group had stronger intensity of VEGF expression compared to the non-endometriosis group who tended to have moderate VEGF intensity. In histoscore calculations, a median value of 4 was obtained in subjects with endometriosis whereas a median value of 3 was obtained in non-endometriosis subjects, and this difference was statistically significant. The results of this study are in conformity with those of Song et al (12), representing that patients with endometriosis had higher VEGF expression compared to subjects in the control group. In the abovementioned study, VEGF expression was known through the immunohistochemical staining of the endometriosis tissue compared to the normal ovarian tissue. High VEGF expression was observed in $69.88 \%$ of subjects who experienced endometriosis compared with $10 \%$ of subjects in the control group, indicating a ratio of 6.9: 1 (higher than the results of this study). VEGF expression levels were also found to be higher in patients with grade III-IV endometriosis compared to those who experienced grade I-II endometriosis. Similar to the current study, the results of another study measuring the histological scores of Von Willebrand factor, alpha-smooth muscle actin, and VEGF in endometriosis models in mice revealed that the number of positive staining cells to VEGF immunocytochemical staining was significantly higher compared to normal endometrium. On the examination of the density of micro blood vessels (microvessels), an increase was found in the number of micro blood vessels in endometriosis stroma when compared to normal endometrium (13). Similar results were also reported in studies that performed immunostaining VEGF on endometrial polyp cells, where a significant increase was detected in VEGF immunopositivity as compared to the control group (14). Regarding the severity of endometriosis, the results of another study evaluating several biomarkers (including interleukin-6, tumor necrosis factor-alpha, matrix metalloproteinase-2, and VEGF) between subjects with stage I-II and III-IV endometriosis demonstrated that only VEGF levels had significant differences between the two groups. The cut-off point for serum VEGF levels that can be used to differentiate between the two stages is at $314.75 \mathrm{pg} / \mathrm{mL}$, where serum VEGF levels above that number indicate a higher degree of endometriosis (stage III or IV).

There are several theories that explain the relationship between endometriosis and VEGF. Endometriosis is an angiogenesis-dependent disease, implying that its level of occurrence is influenced by an increase in the activity of angiogenic factors or a decrease in the activity of angiogenesis inhibitors. The first theory states that a local or exogenous stimulus can cause metaplasia from the mesothelial cell layer in the peritoneal wall. The metaplastic lesion grows by obtaining blood supply after the local invasion of the basement membrane. The second theory indicates that endometriotic cells may systemically spread through blood or lymphatic vessels. The third theory is related to retrograde menstruation, in which viable endometrial cells can ectopically move into the abdomen through the Fallopian tube during menstruation. More than $90 \%$ of women experience retrograde menstruation although the determinants of endometriosis cases in women experiencing retrograde menstruations are influenced by the angiogenic potential of each individual. Women with high angiogenic potentials are more likely to have endometriosis because of their ability to support the tissue growth arising from the retrograde menstruation, causing endometriosis.

VEGF is the most prominent proangiogenic in humans. VEGF expression itself is influenced by various hormones including estrogen and progesterone. Additionally, VEGF is believed to be the main stimulus for angiogenesis in endometriosis and is a growth factor that increases vascular permeability in endometriosis cases. Likewise, VEGF has been found to be elevated in endometrial lesions and the peritoneal fluid in patients with endometriosis, where VEGF levels are the highest in active red endometriotic lesions. The induction of angiogenesis by VEGF is the main and most important pathogenesis in endometriosis, and this phenomenon has a close relationship with the VEGF receptor 2. Various polymorphisms in the VEGF gene are also reported to be associated with an increased risk of endometriosis.

As it was discussed earlier, endometriosis is an angiogenesis-dependent disease the occurrence of which is closely related to the level of angiogenic factors (12). The main mechanism of oxidative stress-induced angiogenesis involves the hypoxia-inducible factor and VEGF signaling. This is not always deleterious since angiogenesis is itself important for maintaining the cyclical regeneration of the endometrium in the menstrual cycle, and this cycle is itself regulated by ROS (15). However, oxidative stress, which is defined as an imbalance between ROS and antioxidants, might be related to the pathophysiology of endometriosis, due to its contribution to causing an inflammatory response in the peritoneal cavity (16). ROS itself is considered as a proinflammatory mediator which 
modulates cell proliferation. The results of a study showed that increased ROS production causes higher oxidative stress levels of endometriotic cells, increasing cellular proliferation (17).

In this study, no significant differences were found in VEGF expression variables between subjects with different pain scales, which is in line with the results of by García-Manero et al, demonstrating no association between pain symptoms in endometriosis patients with serum VEGF levels or VEGF expression in the cells of patients with endometriosis. The absence of a relationship between VEGF and the pain scale may be related to the condition of endometriosis because VEGF expression in other diseases such as osteoarthritis has been found to be associated with pain, where higher expression is found in patients with visual analog scale (VAS) greater than 6 and less than 6 (18). Changes in the expression and VEGF-A signaling profile are thought to be closely related to the onset of chronic pain. VEGF-A can bind to sensory neurons by inducing the outgrowth and survival of sensory neuron axons. VEGF can sensitize C-fiber pain nerves which are sensitive to mechanical pain stimulation, which increases pain signal transmission and mechanical allodynia development. This increased pain sensation is mediated by the activity of the transient receptor potential vanilloid 1 (TRPV1) and TRPA1 canals.

The findings of the current study revealed no difference between VEGF levels and infertility on endometriosis subjects. This finding matches the results of several previous studies. For example, Hafner et al found that VEGF levels in follicular fluid do not correlate with infertility or embryo quality (19). VEGF may have a role in the angiogenic processes of follicular growth and might affect several key events in the ovarian cycle, including follicular development, ovulation, corpus luteum development, and ovarium steroidogenesis. The expression of VEGF itself is regulated by gonadotropins and ovarian sexual hormones $(19,20)$.

Based on the results of this study, no significant relationship was observed regarding the cyst size between the groups of subjects with low and high VEGF expression, which contradicts the findings of Goteri et al, indicating that diffused VEGF expression in endometriosis epithelial cells was associated with cyst sizes larger than $5.4 \mathrm{~cm}$, and high VEGF expression in capsular fibroblasts was associated with bilateral cysts. The findings of another study examining VEGF immunoreactivity in ovarian endometriosis cysts demonstrated positive VEGF immunoreactivity levels in $92 \%$ of samples and indicated that the reactivity was related to cyclooxygenase- 2 levels. The increase in both parameters was statistically related to the increased microvessel density in endometriosis cysts (21). Hazelton et al also found that high levels of VEGF in the cyst fluid were associated with the recurrence and malignancy of cysts (22).

In this study, a significant difference was found in the cyst size between the groups of subjects with mild and moderate VAS, indicating that subjects in the mild VAS group had significantly smaller cyst sizes compared to those in the moderate VAS group. Significant differences were also observed in the cyst size between the groups of subjects with mild VAS and heavily controlled VAS, where subjects in the mild VAS group had significantly smaller cyst sizes in comparison with those in the heavily controlled VAS group. Although the size of the cyst in subjects of the controlled weight VAS group was greater than that of the cyst in subjects in the moderate VAS group, no significant difference was found between the two groups.

Endometriosis is found in quite high numbers in women who experience chronic pain. Approximately $70 \%$ of women experiencing chronic pelvic pain suffer from endometriosis. This is exacerbated by the frequent delay in the diagnosis of endometriosis, which causes a decrease in the quality of life. Chronic pain itself is also one of the symptoms of endometriosis, where patients with endometriosis often complain of pain, including pain during menstruation (dysmenorrhea), sexual intercourse (dyspareunia), defecation (dyschezia), and urination (dysuria). Pain in the pelvis is also often found in short periods before the menstrual period (6).

This study has some limitations. Several subjects were not showing on the sampling day so that they were replaced with other subjects or needed to have their sampling date rescheduled to the next day, which might have affected the results. The use of VAS for measuring pain levels and the use of subjective immunocytochemical measurements might also contribute to several degrees of errors due to the subjective nature of both VAS and immunocytochemical observation. Several other studies in this field should be conducted regarding other substances that might modulate or affect endometriosis, as well as regarding substances that can inhibit the effect of VEGF in order to prevent the occurrence and development of endometriosis.

\section{Conclusions}

VEGF levels in endometriosis women were significantly higher compared to non-endometriosis women. However, it showed no correlation either with pain levels or endometrioma cyst size.

Larger endometriotic cyst sizes were proportionally related to the higher pain levels in subjects with endometriosis. Further research should be done aiming at reducing VEGF levels in subjects with endometriosis. Finally, we also did not find any correlation between VEGF expression, endometriosis, and infertility status in our study.

\section{Authors' Contribution}

RA and Su designed the study and conducted the research. SS, SI, and BHP monitored, evaluated, and analyzed the result of the study. Further, RA, TD, WP, and THM reviewed the article. All authors approved the final manuscript. 


\section{Conflict of Interests}

Authors declare that they have no conflict of interests.

\section{Ethical Issues}

The ethical clearance was reviewed by the Health Research Ethics Committee of Hasan Sadikin Hospital. Permission was obtained from all participating respondents prior to initiating the study. Subject confidentiality was maintained during and after the study.

\section{Financial Support}

This research was funded by the authors themselves. We received no financial support from other external sources.

\section{References}

1. Burney RO, Giudice LC. Pathogenesis and pathophysiology of endometriosis. Fertil Steril. 2012;98(3):511-519. doi:10.1016/j. fertnstert.2012.06.029

2. Dunselman GA, Vermeulen N, Becker C, et al. ESHRE guideline: management of women with endometriosis. Hum Reprod. 2014;29(3):400-412. doi:10.1093/humrep/det457

3. Pangemanan G, Loho M, Wagey F. Profil Penderita Endometriosis di RSUP Prof. Dr. R. D. Kandou Manado pada Tahun 2011-2015. Jurnal eClinic. 2015;5(1):6.

4. Puspasari B, Baziad A, Hestiantoro A. Karakteristik Pasien Endometriosis di Rumah Sakit Dr. Cipto Mangunkusumo Selama Periode 1 Januari 2000 - 31 Desember 2005 Majalah Obstetri dan Ginekologi Indonesia. 2007;31(2):6.

5. Rahmawati DS. Gambaran Karakteristik dan Pencarian Pelayanan Kesehatan pada Penderita Endometriosis di Klinik Fertilitas Graha Amerta RSUD dr. Soetomo, Surabaya. Surabaya: Universitas Airlangga; 2016.

6. Parasar P, Ozcan P, Terry KL. Endometriosis: epidemiology, diagnosis and clinical management. Curr Obstet Gynecol Rep. 2017;6(1):34-41. doi:10.1007/s13669-017-0187-1

7. Pretta S, Remorgida V, Abbamonte LH, et al. Atherosclerosis in women with endometriosis. Eur J Obstet Gynecol Reprod Biol. 2007;132(2):226-231. doi:10.1016/j.ejogrb.2006.04.015

8. Haas D, Chvatal R, Reichert B, Renner S, Shebl O, Binder H, et al. Endometriosis: a premenopausal disease? Age pattern in 42,079 patients with endometriosis. Arch Gynecol Obstet. 2012;286(3):667-70.

9. Yamamoto A, Johnstone EB, Bloom MS, Huddleston HG, Fujimoto VY. A higher prevalence of endometriosis among Asian women does not contribute to poorer IVF outcomes. J Assist Reprod Genet. 2017;34(6):765-774. doi:10.1007/s10815-017-0919-1

10. Jenkins J, Holt V. Endometriosis. In: Goldman MB, Hatch MC, eds. Women and Health. New York: Academic Press; 2000:226-239.
11. Shah DK, Correia KF, Vitonis AF, Missmer SA. Body size and endometriosis: results from 20 years of follow-up within the Nurses' Health Study II prospective cohort. Hum Reprod. 2013;28(7):1783-1792. doi:10.1093/humrep/det120

12. Song WW, Lu H, Hou WJ, et al. Expression of vascular endothelial growth factor $\mathrm{C}$ and anti-angiogenesis therapy in endometriosis. Int J Clin Exp Pathol. 2014;7(11):7752-7759.

13. Machado DE, Berardo PT, Palmero CY, Nasciutti LE. Higher expression of vascular endothelial growth factor (VEGF) and its receptor VEGFR-2 (Flk-1) and metalloproteinase-9 (MMP-9) in a rat model of peritoneal endometriosis is similar to cancer diseases. J Exp Clin Cancer Res. 2010;29(1):4. doi:10.1186/1756-9966-294

14. Erinanc $\mathrm{H}$, Toprak E. Increased expression of nestin and VEGF in endometrial polyps: an immunhistochemical study. Int J Clin Exp Pathol. 2016;9(7):7458-7463.

15. Kim YW, Byzova TV. Oxidative stress in angiogenesis and vascular disease. Blood. 2014;123(5):625-631. doi:10.1182/ blood-2013-09-512749

16. Scutiero G, lannone P, Bernardi G, et al. Oxidative stress and endometriosis: a systematic review of the literature. Oxid Med Cell Longev. 2017;2017:7265238. doi:10.1155/2017/7265238

17. Ngô C, Chéreau C, Nicco C, Weill B, Chapron C, Batteux F. Reactive oxygen species controls endometriosis progression. Am J Pathol. 2009;175(1):225-234. doi:10.2353/ajpath.2009.080804

18. García-Manero M, Alcazar JL, Toledo G. Vascular endothelial growth factor (VEGF) and ovarian endometriosis: correlation between VEGF serum levels, VEGF cellular expression, and pelvic pain. Fertil Steril. 2007;88(2):513-515. doi:10.1016/j. fertnstert.2006.11.117

19. Hafner D, Zivkovic SV, Bauman R, Tiljak K, Papić N, Lepej SZ. Follicular fluid vascular endothelial growth factor is associated with type of infertility and interferon alpha correlates with endometrial thickness in natural cycle in vitro fertilization. Reprod Biol. 2018;18(3):289-294. doi:10.1016/j.repbio.2018.06.001

20. Lam PM, Haines C. Vascular endothelial growth factor plays more than an angiogenic role in the female reproductive system. Fertil Steril. 2005;84(6):1775-1778. doi:10.1016/j. fertnstert.2005.05.059

21. Goteri G, Lucarini G, Filosa A, et al. Immunohistochemical analysis of vascular endothelial growth factor cellular expression in ovarian endometriomata. Fertil Steril. 2004;81(6):1528-1533. doi:10.1016/j.fertnstert.2003.11.035

22. Hazelton D, Nicosia RF, Nicosia SV. Vascular endothelial growth factor levels in ovarian cyst fluid correlate with malignancy. Clin Cancer Res. 1999;5(4):823-829.

(C) 2021 The Author(s); This is an open-access article distributed under the terms of the Creative Commons Attribution License (http:// creativecommons.org/licenses/by/4.0), which permits unrestricted use, distribution, and reproduction in any medium, provided the original work is properly cited. 